Review Article

\title{
Feeding Practices among Human Immunodeficiency Virus-Exposed Infants in Ethiopia: Systematic Review and Meta-Analysis
}

\author{
Habtamu Temesgen (D, ${ }^{1}$ Ayenew Negesse, ${ }^{1}$ Temesgen Getaneh, ${ }^{2}$ Yibelu Bazezew, ${ }^{2}$ \\ Dessalegn Haile $\left(\mathbb{1},{ }^{3}\right.$ Wubetu Woyraw, ${ }^{1}$ Melaku Desta, ${ }^{2}$ and Alehegn Aderaw Alamneh ${ }^{1}$ \\ ${ }^{1}$ Department of Human Nutrition and Food Science, College of Health Science, Debre Markos University, Debre Markos, Ethiopia \\ ${ }^{2}$ Department of Midwifery, College of Health Science, Debre Markos University, Debre Markos, Ethiopia \\ ${ }^{3}$ Department of Nursing, College of Health Science, Debre Markos University, Debre Markos, Ethiopia
}

Correspondence should be addressed to Habtamu Temesgen; habtamutem@gmail.com

Received 27 February 2020; Revised 10 June 2020; Accepted 25 February 2021; Published 10 March 2021

Academic Editor: Hamidreza Karimi-Sari

Copyright (c) 2021 Habtamu Temesgen et al. This is an open access article distributed under the Creative Commons Attribution License, which permits unrestricted use, distribution, and reproduction in any medium, provided the original work is properly cited.

\begin{abstract}
Background. Infant and young child feeding in the context of human immunodeficiency virus- (HIV-) infected mothers has significant challenges due to the risk of transmission of the virus via breastfeeding. In Ethiopia, a number of independent studies have been conducted to assess the feeding practice of HIV-exposed infants. But, there is no concrete evidence to show the national figure in Ethiopia. Hence, this review and meta-analysis aims to estimate the pooled prevalence of feeding practices among HIV exposed infants in Ethiopia. Methods. The Preferred Reporting Items for Systematic Reviews and Meta-Analyses (PRISMA) guideline was followed. Articles were searched through search engines in PubMed, Cochrane Library, Google Scholar, and direct Google search. Joanna Briggs Institute Meta-Analysis of Statistics Assessment and Review Instrument adapted for cross-sectional study design was used for quality assessment. The random effects model was used to estimate the pooled prevalence of infant feeding practices. Heterogeneity and publication bias were assessed. Trim and fill analysis was performed. Additionally, meta-regression was also performed. Results. In this review, a total of 26, 22, and 22 studies and 7413, 6224, and 6222 study participants for exclusive breastfeeding, replacement feeding, and mixed feelings were included, respectively. The overall pooled prevalence of exclusive breastfeeding, replacement feeding, and mixed feeding of HIV exposed infant was $63.99 \%$ (95\% Confidence Interval (CI): 52.32, 75.66), 16.13\% (95\% CI: 11.92, 20.32), and 20.95\% (95\% CI: 11.35, 30.58)) in Ethiopia, respectively. Conclusion and Recommendations. In Ethiopia, almost three in five HIV-exposed infants were exclusively breastfed. But still, mixed feeding during the period of first 6 months was practiced in almost one-fifth of the exposed infants in Ethiopia. Additionally, replacement feeding was also practiced even though not recommended for developing countries. Therefore, the government of Ethiopia should strengthen the health institutions to implement the existing infant feeding strategies and guidelines to increase exclusive breastfeeding for the first 6 months and to avoid mixed feeding during the periods of six months.
\end{abstract}

\section{Background}

Infant feeding, especially during the first years of life, is a major determinant of child survival and development. Even though it contributes about $15 \%$ HIV transmission, breastfeeding is universal socioculturally acceptable, nutritious way to feed and provide immunity to the infants [1].
Infant and young child feeding in the context of HIVinfected mother has significant challenges due to the risk of virus transmission through breastfeeding [2]. It should support the infants to be free from HIV and does not harm the health of the mothers [2]. Globally, an estimated 43,0000 children were newly infected with HIV in 2008; the majority of them were infected through 
mother-to-child transmission (MTCT) $[3,4]$. Perinatal periods contribute to HIV infection in about $15 \%$ to $35 \%$ infants if there is no intervention from the infected mothers $[5,6]$ and about 10 to $20 \%$ are infected through breast milk during breastfeeding [7].

Before 2010, avoidance or early cessation of breastfeeding was recommended. However, the consequences for the health and survival of the infants were serious, with much higher mortality rates due to diarrhea, malnutrition, and other diseases in non-breastfed children. Due to these risks, the World Health Organization after 2010 recommends every country should decide the best option of infant feeding practices and interventions. Even though the World Health Organization (WHO) suggests different infant feeding strategies, exclusive breastfeeding is recommended for the first six months in developing countries [2]. Replacement feeding for HIV exposed infant should not be used unless is it acceptable, feasible, affordable, sustainable, and safe (AFASS) $[2,8]$.

The method of infant feeding is clearly associated with the risk of transmission through breast milk. Exclusive breastfeeding for the first six months is associated with a 3-4 fold lower risk of HIV transmission as compared to mixed feeding [9]. About $4 \%$ of exclusively breastfed infants became infected with HIV between 6 weeks and 6 months, even in the absence of Anti-Retroviral Treatment (ART) [9]. Currently, mixed feeding is still the norm in developing countries for HIV-exposed infants less than six months and carries a greater risk of HIV transmission [10]. Exclusive breastfeeding rates among children less than 6 months of age in two-thirds of developing countries is decreasing, which is quite low (33\%) in sub-Saharan Africa [11]. HIV transmission through breastfeeding can be reduced if HIVpositive women breastfeed exclusively for six months rather than practicing mixed feeding and are strongly encouraged to continue breastfeeding [3].

In Ethiopia, a number of different discrete and independent researches were conducted to assess the feeding practices of HIV-exposed infants [12-36]. These independent studies described that the feeding practices of HIVexposed infants in Ethiopia were ranging from $18.1 \%$ up to $96.6 \%$ exclusive breastfeeding [20,24], $0.5 \%$ to $46.8 \%$ replacement feeding $[12,20,30]$, and $3.7 \%$ to $81 \%$ mixed feeding $[20,24]$. An individual study conducted in Ethiopia revealed that the prevalence of infant feeding practices among HIV exposed infants is inconsistent across regions, and there is a variation over time. Even though a metaanalysis was done in Ethiopia [37], it does not include the study on replacement feeding and it missed the number of individual. Therefore, this review and meta-analysis aimed to estimate the pooled prevalence of feeding practices of HIV-exposed infants in Ethiopia with including replacement feeding and the missed articles.

The findings of this study will be an input to program planners of the government of Ethiopia to allocate appropriate resources and design appropriate interventions to increase exclusive breastfeeding and reduce replacement feeding and mixed feeding practice among HIV-exposed infants.

\section{Methods}

2.1. Searching Strategies. First, to avoid duplications, databases were searched for the same systematic review and meta-analysis in Ethiopia on infant feeding practices among HIV-exposed infants. We can confirm that there were no similar review and meta-analysis on this topic in Ethiopia.

Published and unpublished researches were reviewed to estimate the pooled prevalence of infant feeding practices in Ethiopia. The major databases such as PUBMED/MEDLINE, Cochrane library, Google, and Google Scholar were used to identify the published research. Whereas unpublished researches were found from institution websites and direct Google searches. The key term used in PubMed search was "Prevalence" OR "infant feeding" OR "exclusive breastfeeding," "replacement feeding", "mixed feeding," "HIV-exposed infant," AND "Ethiopia". Medical subject headings (MeSH terms) were used to help expand the search in the advanced PubMed search. This study also used the Boolean operator (within each axis, we combined the keywords with the "OR" operator and we then linked the search strategies for the two axes with the "AND" operator). We followed the Preferred Reporting Items for Systematic Reviews and Meta-Analyses (PRISMA) guideline during the systematic review [38].

\subsection{Inclusion Criteria}

2.2.1. Study Scope. All studies which report the prevalence of exclusive breastfeeding, replacement feeding practice, and mixed feeding practices among HIV-Exposed in Ethiopia was included. Study area: researches were conducted only in Ethiopia.

2.2.2. Study Design. By default, cross-sectional study designs were included.

2.2.3. Language. All articles written in English language were included.

2.2.4. Population. Studies conducted among HIV-exposed infants feeding practices in Ethiopia were included.

2.2.5. Study Year. Only articles studied until June 2020 were included.

2.2.6. Publications. Both published and unpublished articles were included.

2.3. Exclusion Criteria. Those studies, which were not fully retrieved after reading the titles, were excluded. Studies, which did not report infant feeding practices (at least one), were excluded after reviewing their full texts. 
2.3.1. Abstraction. Data were extracted by two authors (TG and HT) using a standardized data extraction excel spread sheet. The data extraction format includes the primary author, study year, publication year, region of study, sample size, prevalence of exclusive breastfeeding, prevalence of replacement feeding practices, and mixed feeding practices.

2.3.2. Quality Assessment. First, the database search results were combined, and duplicate articles were removed manually using Endnote (version X 7.2). Joanna Briggs Institute MetaAnalysis of Statistics Assessment and Review Instrument adapted for the prevalence study design was used for quality assessment [39]. Each individual paper was assessed by two independent reviewers (TG and YB). Discrepancies between those reviewers were solved by discussion. If not, others were involved to resolve inconsistencies in between the two independent reviewers. Studies which scored $50 \%$ and above were included in the final review and analysis.

2.4. Outcome Measurements. This review and meta-analysis has one main outcome: the estimated pooled prevalence of infant feeding practices (exclusive breast feeding, replacement feeding, and mixed feeding practices) among HIVexposed infant in Ethiopia.

2.4.1. Replacement Feeding. It is feeding an infant, who is not receiving breast milk, with a diet that provides all the nutrients children need until they can be fully fed on family foods [40].

2.4.2. Exclusive Breastfeeding. The infant receives only breast milk without any other liquids or solids, not even water, except for oral rehydration solution or drops or syrups of vitamins, minerals, or medicines [40].

2.4.3. Mixed Feeding. An infant younger than six months of age is given other liquids and/or food together with breast milk [40].

2.5. Data Analysis. Firstly, the data extraction was done by using excel sheet, and then the excel data were saved as CSV (delimited, comma) and then imported to STATA 14 for analysis. To estimate the pooled prevalence, first, the standard error of each individual studies was generated. After generating the standard errors for each prevalence value, the pooled prevalence was reported with 95\% CI. Heterogeneity across studies was assessed by using the inverse variance $\left(I^{2}\right)$ at $25 \%$, $50 \%$, and $75 \%$ as low, moderate, and considerable heterogeneity, respectively, with a $p$ value less than 0.05 [41]. Due to the presence of heterogeneity (by fixed effects model), the random effects meta-analysis model was used to estimate the pooled prevalence of infant feeding practices among HIVexposed infants in Ethiopia. To visualize the presence of heterogeneity subjectively, the forest plot also used. Subgroup analysis was conducted to identify the source of heterogeneity. Also, publication bias was assessed using both Egger's and
Begg's tests at $p$ value less than $0.05[42,43]$. Trim and fill meta-analysis [44] has been done to see and adjust the influence of publication bias. Finally, meta-regression was performed to show the association between the selected variables with heterogeneity of the pooled prevalence of infant feeding practices and declare the statistically significance at $p$ value less than 0.05 .

\section{Results}

3.1. Selection of Studies. A total of 398 studies were retrieved through electronic searching. From these, 140 duplicated records were excluded, and from 258 articles screened using their titles and abstracts, 232 were excluded. Twenty-six fulltext articles were assessed for eligibility. All the 26 studies were included in the final systematic review and metaanalysis, but only 22 were included for mixed and replacement feedings (Figure 1).

3.2. Characteristics of Included Studies. In this systematic review and meta-analysis, five regions and one town administrative in Ethiopia were represented. From the total studies, seven were from the Amhara region $[15,17,18,21,31,34,45]$, six were from Addis Ababa $[13,14,16,27,29,30]$, six were from Oromia [12, 20, 23, 32, 33, 35], four were from South Nations Nationalities and People (SNNP) [19, 24-26], and three were from Tigray region $[22,28,36]$. From the total studies, twenty-one studies were published, while five studies were unpublished (Table 1). All the included studies used crosssectional study design. Twenty-four studies were institution based while two studies were community based (Table 1). The sample size of the included studies ranged from 87 [25] to 657 [19]. In this review, a total of 7413, 6224, and 6222 study participants were included to estimate the pooled prevalence of exclusive breastfeeding replacement feeding and mixed feeding practices in Ethiopia (Table 1).

From the total studies, four studies did not report the prevalence of replacement feeding $[14,16,18,23]$ and mixed feeding practices $[14,16,18,27]$ of HIV-exposed infant in Ethiopia. Five studies used nonprobability sampling techniques, while the twenty-one studies used probability sampling techniques (Table 1).

3.3. Pooled Prevalence of Infant Feeding Practices of HIVExposed Infant in Ethiopia (Meta-Analysis). The pooled prevalence of exclusive breastfeeding practices among HIV exposed infant in Ethiopia was 63.99 \% (95\% CI: 52.32, 75.66). The random effects model was used to estimate the pooled prevalence of infant feeding practices. Both graphically and statistically significant heterogeneity was observed $\left(I^{2}=99.4 \% ; P<0.001\right)$ (Figure 2). The pooled prevalence of replacement and mixed feeding practices of HIV-exposed infants was $16.13 \%(95 \%$ CI: 11.94, 20.31) and 20.95\% (95\% CI: $11.35,30.58)$ with severe heterogeneity $\left(I^{2}=98.9 \%\right.$; $P<0.001)$ and $\left(I^{2}=99.3 \% ; P<0.001\right.$ (Figures 3 and 4$)$, respectively. The presence of significant heterogeneity leads to conduct subgroup analysis to identify the sources of heterogeneity. 


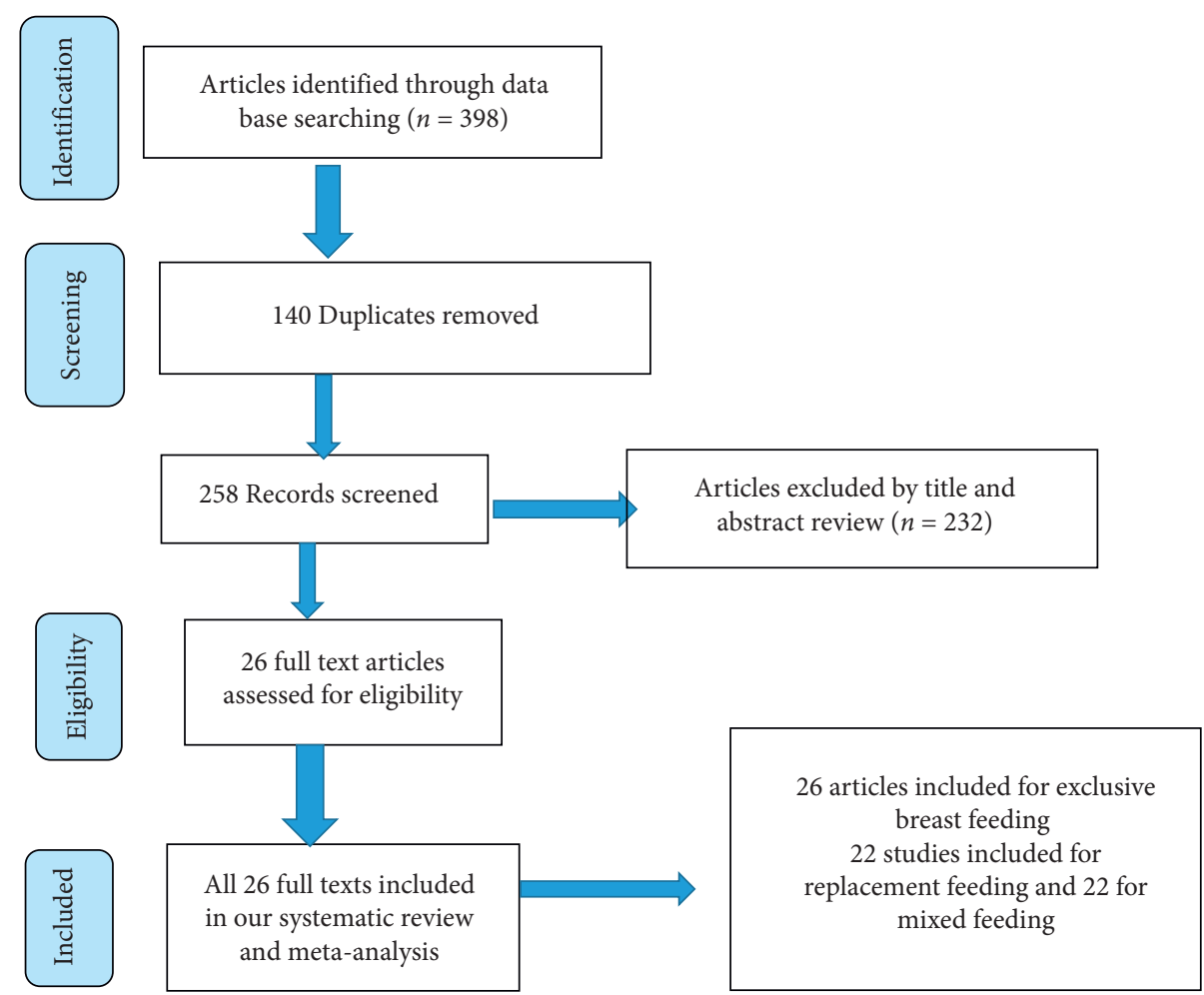

FIGURE 1: PRISMA flow diagram of included studies to estimate the pooled prevalence of HIV-exposed infant feeding practices in Ethiopia.

3.4. Subgroup Analysis. In this meta-analysis, there is a significant heterogeneity across studies among HIV-exposed infant feeding options in Ethiopia (Figures 2, 3, and 4). Therefore, subgroup analysis based on regions of the study, study years, and sampling techniques was performed to identify the possible source of heterogeneity. The results indicated that the source of heterogeneity was not due to regions, study years, and sampling techniques.

The highest prevalence of exclusive breastfeeding practice was observed in Tigray region, $89.75 \%(87.56$, 91.95) followed by Amhara region, 72.84\% (62.41, 83.28), $\left.I^{2}=96.8 \%, P<0.001\right)$. The pooled prevalence of exclusive breastfeeding was $58.75 \%(42.69,74.82)$ before the year of 2015 and $71.21 \%(59.19,83.22) 2015$ and after 2015 (Table 2). The studies conducted by using convenient (consecutive) sampling techniques relatively had higher pooled prevalence of exclusive feeding practice $71.26 \%$ $(43.26,99.26)$ (Table 2).

Replacement feeding was highly practiced in Addis Ababa $23.80 \%(7.95,39.66)$ while the lowest prevalence was in Tigray region $(3.92 \%(2.52,5.33))$ and Oromia $(13.27 \%$ $(5.75,20.79)$ region. Additionally, lowest prevalence of replacement feeding was indicated in studies that were used random sampling techniques $(11.47 \%(4.48,18.46)$. There is no significant difference in pooled prevalence of replacement feeding before and after 2015 years (Table 2).

Mixed feeding was more prevalent in Addis Ababa (40.03\%) followed by Oromia region $(26.46 \%)$ while the lowest prevalence was seen in Tigray regions (6.29\%). There was significant decrement of mixed feeding practices starting from $2015(8.98 \%(4.19,13.77))$ (Table 2).
Even though the subgroup analysis could not decrease the level of heterogeneity, substantial variation of infant feeding practices was observed among regions, study year, and sampling techniques.

3.5. Publication Bias. Publication bias was also checked using funnel plot statistically by Begg's and egger's tests [42, 43]. Egger's and Begg's tests $(p$-value $<0.05)$ indicated that there was publication bias for replacement feeding practice among HIV-exposed infants. Additionally, the funnel plot indicates the presence of publication bias of replacement feeding (Figure 5). Therefore, Trim and fill meta-analysis [44] was done to explain the publication bias of replacement feeding practice. The prevalence of replacement feeding practice among HIV-exposed infants after adjusting by using trim-fill analysis was similar (16.13\%) to the pooled prevalence of replacement feeding practices. Therefore, no significant change was reported. Egger's and Begg's tests also indicated that the publication bias was not observed for exclusive breastfeeding $(P$ value $>0.05)$ and mixed feeding practices $(P$ value $>0.05)$. Also, there is no publication bias for exclusive breastfeeding and mixed feeding practice by using funnel plots (Figures 6 and 7).

3.6. Sensitivity Analysis. Sensitivity analysis was checked to identify the outliers of the studies for EPF, replacement, and mixed feeding practices. There was not any individual study which influences the overall pooled estimate of exclusive breast feeding. But, Yetayesh et al., Tadelech Mebratu, Chernet et al., and Tefera et al. influenced the pooled 
TABLE 1: . Characteristics of 26 included studies to estimate the pooled prevalence of exclusive breastfeeding and 24 studies for replacement feeding and mixed feeding practices of HIV-exposed infants in Ethiopia.

\begin{tabular}{|c|c|c|c|c|c|c|c|c|c|c|c|}
\hline S.no & Authors & $\begin{array}{l}\text { Study } \\
\text { region }\end{array}$ & $\begin{array}{l}\text { Study } \\
\text { years }\end{array}$ & $\begin{array}{c}\text { Publication } \\
\text { years }\end{array}$ & $\begin{array}{l}\text { Sample } \\
\text { size }\end{array}$ & $\begin{array}{l}\text { Study } \\
\text { design }\end{array}$ & $\begin{array}{l}\text { Study } \\
\text { setting }\end{array}$ & $\begin{array}{l}\text { Sampling } \\
\text { technique }\end{array}$ & $\begin{array}{c}\text { Prevalence } \\
\text { EBF }\end{array}$ & $\begin{array}{c}\text { Prevalence } \\
\text { RF }\end{array}$ & $\begin{array}{c}\text { Prevalence } \\
\text { MF }\end{array}$ \\
\hline 1 & $\begin{array}{c}\text { Mengistu } \\
\text { and Kedir } \\
{[12]}\end{array}$ & Oromia & 2016 & 2017 & 327 & $\begin{array}{c}\text { Cross } \\
\text { sectional }\end{array}$ & Institution & $\begin{array}{c}\text { Simple } \\
\text { random } \\
\text { sampling }\end{array}$ & 30.6 & 46.8 & 15.3 \\
\hline 2 & $\begin{array}{c}\text { Mukerem } \\
\text { M and } \\
\text { Haidar J } \\
{[14]}\end{array}$ & $\begin{array}{l}\text { Addis } \\
\text { ababa }\end{array}$ & 2012 & 2012 & 348 & $\begin{array}{c}\text { Cross } \\
\text { sectional }\end{array}$ & Institution & Consecutive & 73 & - & - \\
\hline 3 & $\begin{array}{c}\text { Sendo et al } \\
{[15]}\end{array}$ & Amhara & 2017 & 2018 & 230 & $\begin{array}{c}\text { Cross } \\
\text { sectional }\end{array}$ & Institution & $\begin{array}{c}\text { Systematic } \\
\text { random }\end{array}$ & 75.2 & 13.9 & 10.9 \\
\hline 4 & $\begin{array}{c}\text { Demelew } \\
\text { MZ and } \\
\text { Abdeta G } \\
{[16]}\end{array}$ & $\begin{array}{l}\text { Addis } \\
\text { ababa }\end{array}$ & 2011 & 2014 & 356 & $\begin{array}{c}\text { Cross } \\
\text { sectional }\end{array}$ & Institution & $\begin{array}{l}\text { Systematic } \\
\text { random }\end{array}$ & 68 & - & - \\
\hline 5 & $\begin{array}{l}\text { Muluye } \\
\text { et al [17] }\end{array}$ & Amhara & 2011 & 2012 & 209 & $\begin{array}{c}\text { Cross } \\
\text { sectional }\end{array}$ & Institution & $\begin{array}{l}\text { Simple } \\
\text { random }\end{array}$ & 83.8 & 5.7 & 10.5 \\
\hline 6 & $\begin{array}{l}\text { Genetu } \\
\text { et al [18] }\end{array}$ & Amhara & 2016 & 2017 & 367 & $\begin{array}{c}\text { Cross } \\
\text { sectional }\end{array}$ & Institution & $\begin{array}{l}\text { Systematic } \\
\text { random }\end{array}$ & 86.4 & - & - \\
\hline 7 & $\begin{array}{c}\text { Tefera } \\
\text { B. and } \\
\text { Challi [19] }\end{array}$ & SNNP & 2004 & 2007 & 657 & $\begin{array}{c}\text { Cross } \\
\text { sectional }\end{array}$ & Community & $\begin{array}{l}\text { Systematic } \\
\text { random }\end{array}$ & 16 & 61 & 16 \\
\hline 8 & $\begin{array}{l}\text { Chernet } \\
\text { Hailu [20] }\end{array}$ & Oromia & 2005 & - & 643 & $\begin{array}{c}\text { Cross } \\
\text { sectional }\end{array}$ & Community & Cluster & 18.1 & 0.6 & 81 \\
\hline 9 & $\begin{array}{l}\text { Wakwoya } \\
\text { et al [21] }\end{array}$ & Amhara & 2013 & 2016 & 260 & $\begin{array}{c}\text { Cross } \\
\text { sectional }\end{array}$ & Institution & $\begin{array}{l}\text { Simple } \\
\text { random }\end{array}$ & 77.3 & 8.5 & 14.2 \\
\hline 10 & $\begin{array}{c}\text { Girma } \\
\text { et al [22] }\end{array}$ & Tigray & 2013 & 2014 & 207 & $\begin{array}{c}\text { Cross } \\
\text { sectional }\end{array}$ & Institution & $\begin{array}{l}\text { Systematic } \\
\text { random }\end{array}$ & 90.3 & 3.4 & 6.3 \\
\hline 11 & $\begin{array}{l}\text { Bekere } \\
\text { et al [23] }\end{array}$ & Oromia & 2014 & 2014 & 118 & $\begin{array}{c}\text { Cross } \\
\text { sectional }\end{array}$ & Institution & $\begin{array}{c}\text { Systematic } \\
\text { random }\end{array}$ & 80.5 & - & 19.5 \\
\hline 12 & $\begin{array}{l}\text { Demissie } \\
\text { et al [24] }\end{array}$ & SNNP & 2016 & 2016 & 146 & $\begin{array}{c}\text { Cross } \\
\text { sectional }\end{array}$ & Institution & Consecutive & 96.6 & 2.7 & 3.7 \\
\hline 13 & $\begin{array}{c}\text { Maru Y } \\
\text { and } \\
\text { Haidar J } \\
\text { [13] }\end{array}$ & $\begin{array}{l}\text { Addis } \\
\text { ababa }\end{array}$ & 2008 & 2009 & 327 & $\begin{array}{c}\text { Cross } \\
\text { sectional }\end{array}$ & Institution & Consecutive & 30.6 & 46.8 & 15.3 \\
\hline 14 & $\begin{array}{c}\text { Mengstie } \\
\text { A. and } \\
\text { Tadese T } \\
{[25]}\end{array}$ & SNNP & 2011 & 2016 & 87 & $\begin{array}{c}\text { Cross } \\
\text { sectional }\end{array}$ & Institution & $\begin{array}{l}\text { Random } \\
\text { sampling }\end{array}$ & 56.3 & 8.1 & 35.6 \\
\hline 15 & $\begin{array}{c}\text { Modjo KE. } \\
\text { and } \\
\text { Amanta } \\
\text { NW [26] }\end{array}$ & SNNP & 2013 & 2015 & 436 & $\begin{array}{c}\text { Cross } \\
\text { sectional }\end{array}$ & Institution & Consecutive & 48.2 & 12.8 & 34.6 \\
\hline 16 & $\begin{array}{c}\text { Wondie } \\
\text { T. and } \\
\text { Worku B } \\
{[27]}\end{array}$ & $\begin{array}{l}\text { Addis } \\
\text { ababa }\end{array}$ & 2010 & - & 116 & $\begin{array}{c}\text { Cross } \\
\text { sectional }\end{array}$ & Institution & $\begin{array}{l}\text { Systematic } \\
\text { random }\end{array}$ & 56 & 44 & - \\
\hline 17 & $\begin{array}{c}\text { Ali Y. and } \\
\text { Reddy PS } \\
{[45]}\end{array}$ & Amhara & 2012 & 2015 & 373 & $\begin{array}{c}\text { Cross } \\
\text { sectional }\end{array}$ & Institution & $\begin{array}{l}\text { Systematic } \\
\text { random }\end{array}$ & 74.8 & 13.4 & 11.8 \\
\hline 18 & $\begin{array}{l}\text { Gejo NG } \\
\text { et al [36] }\end{array}$ & Tigray & 2016 & 2019 & 304 & $\begin{array}{c}\text { Cross } \\
\text { sectional }\end{array}$ & Institution & $\begin{array}{l}\text { Systematic } \\
\text { random }\end{array}$ & 88.8 & 4.6 & 6.6 \\
\hline 19 & $\begin{array}{l}\text { Amdom } \\
\text { G/Hiwot } \\
\text { et al [28] }\end{array}$ & Tigray & 2014 & 2014 & 219 & $\begin{array}{c}\text { Cross } \\
\text { sectional }\end{array}$ & Institution & $\begin{array}{l}\text { Systematic } \\
\text { random }\end{array}$ & 90.4 & 3.7 & 5.9 \\
\hline 20 & $\begin{array}{l}\text { Negash } \\
\text { et al [29] }\end{array}$ & $\begin{array}{l}\text { Addis } \\
\text { ababa }\end{array}$ & 2018 & 2019 & 348 & $\begin{array}{c}\text { Cross } \\
\text { sectional }\end{array}$ & Institution & $\begin{array}{l}\text { Systematic } \\
\text { random }\end{array}$ & 70.7 & 6 & 23.3 \\
\hline
\end{tabular}


TABLE 1: Continued.

\begin{tabular}{|c|c|c|c|c|c|c|c|c|c|c|c|}
\hline S.no & Authors & $\begin{array}{l}\text { Study } \\
\text { region }\end{array}$ & $\begin{array}{l}\text { Study } \\
\text { years }\end{array}$ & $\begin{array}{c}\text { Publication } \\
\text { years }\end{array}$ & $\begin{array}{c}\text { Sample } \\
\text { size }\end{array}$ & $\begin{array}{l}\text { Study } \\
\text { design }\end{array}$ & $\begin{array}{c}\text { Study } \\
\text { setting }\end{array}$ & $\begin{array}{l}\text { Sampling } \\
\text { technique }\end{array}$ & $\begin{array}{c}\text { Prevalence } \\
\text { EBF }\end{array}$ & $\begin{array}{c}\text { Prevalence } \\
\text { RF } \\
\end{array}$ & $\begin{array}{c}\text { Prevalence } \\
\text { MF }\end{array}$ \\
\hline 21 & $\begin{array}{c}\text { Tadelech } \\
\text { Mebratu } \\
{[30]}\end{array}$ & $\begin{array}{l}\text { Addis } \\
\text { ababa }\end{array}$ & 2014 & - & 313 & $\begin{array}{c}\text { Cross } \\
\text { sectional }\end{array}$ & Institution & $\begin{array}{l}\text { Random } \\
\text { sampling }\end{array}$ & 18 & 0.5 & 81.5 \\
\hline 22 & $\begin{array}{c}\text { Dagnew } \\
\mathrm{AB} \text { et al } \\
{[31]}\end{array}$ & Amhara & 2018 & 2019 & 213 & $\begin{array}{c}\text { Cross } \\
\text { sectional }\end{array}$ & Institution & $\begin{array}{l}\text { Systematic } \\
\text { random }\end{array}$ & 36.2 & 47.4 & 16.4 \\
\hline 23 & $\begin{array}{c}\text { Ejara et al } \\
{[32]}\end{array}$ & Oromia & 2016 & 2018 & 283 & $\begin{array}{c}\text { Cross } \\
\text { sectional }\end{array}$ & Institution & $\begin{array}{l}\text { Random } \\
\text { sampling }\end{array}$ & 83.5 & 1.8 & 8.3 \\
\hline 24 & $\begin{array}{c}\text { Zelalem } \\
\text { Ketema } \\
\text { [33] }\end{array}$ & Oromia & 2016 & - & 190 & $\begin{array}{c}\text { Cross } \\
\text { sectional }\end{array}$ & Institution & Consecutive & 84.7 & 3.2 & 6.3 \\
\hline 25 & $\begin{array}{c}\text { Fikir } \\
\text { Tadese } \\
{[34]}\end{array}$ & Amhara & 2017 & - & 230 & $\begin{array}{c}\text { Cross } \\
\text { sectional }\end{array}$ & Institution & $\begin{array}{l}\text { Systematic } \\
\text { random }\end{array}$ & 75.2 & 13.9 & 10.9 \\
\hline 26 & $\begin{array}{l}\text { Kefyalew } \\
\text { Taye } \\
\text { Belete [35] }\end{array}$ & Oromia & 2019 & 2020 & 106 & $\begin{array}{c}\text { Cross } \\
\text { sectional }\end{array}$ & Institution & $\begin{array}{l}\text { Systematic } \\
\text { random }\end{array}$ & 53.8 & 17.9 & 28.3 \\
\hline
\end{tabular}

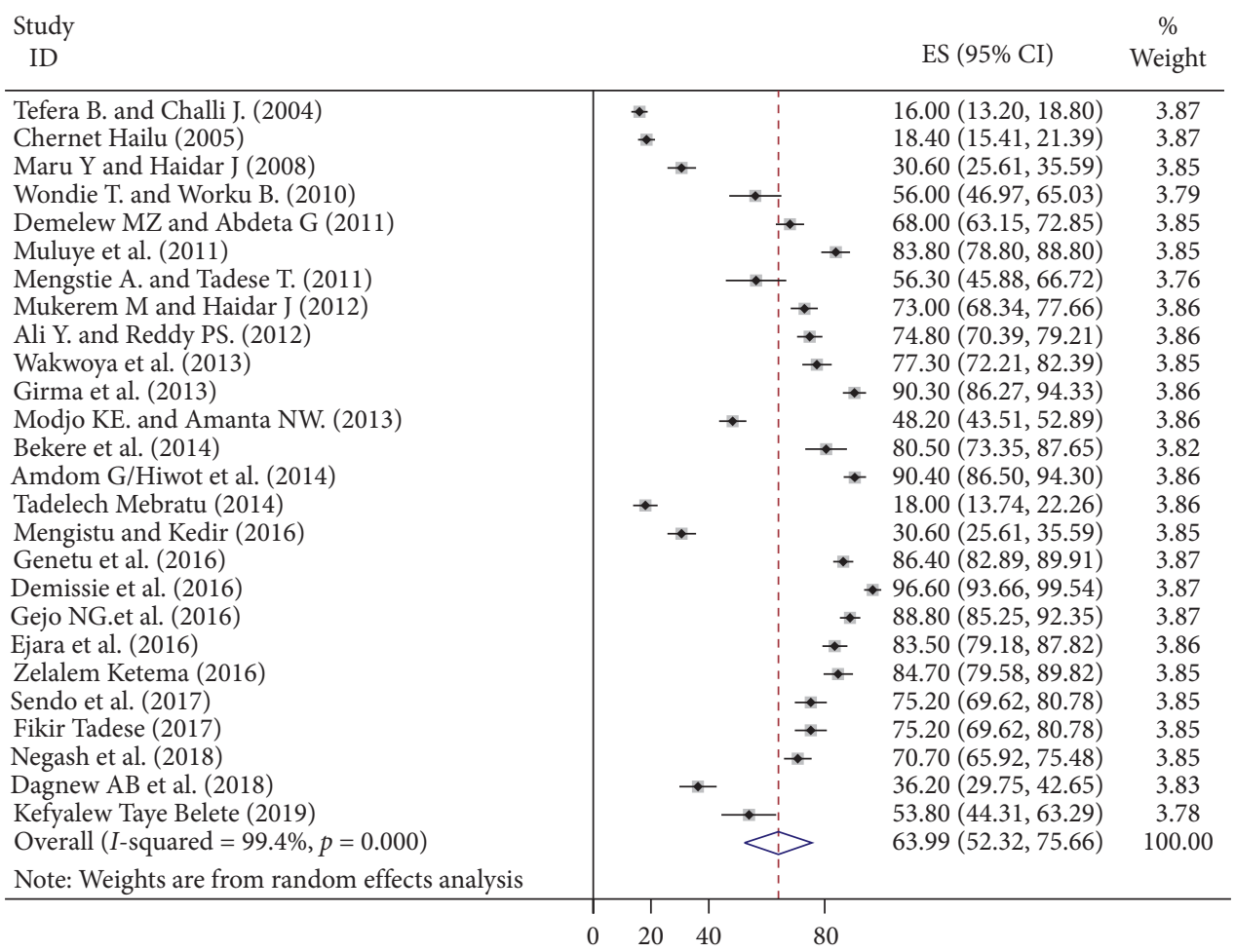

FIGURE 2: Forest plot showing the pooled prevalence of exclusive breastfeeding practice of HIV-exposed infants in Ethiopia.

prevalence of replacement feeding. Additionally, mixed feeding was influenced by Tadelech Mebratu.

3.7. Meta Regression. Meta-regression was performed by considering both continuous and categorical data to identify factors of heterogeneity for the pooled prevalence infant feeding practices among HIV-exposed infants. Sample size, study year, and study area (regions) were considered to perform meta-regression. But, the result of meta-regression showed that the pooled prevalence of infant feeding practices was not associated with study area and study years. However, sample size was associated with exclusive breastfeeding practices ( $P$ value 0.007 ) (Table 3 ). So, subgroup analysis was done to see the variations, and it indicated that the pooled prevalence was decreased when the sample size was 384 and above $(27.42 \%(10.96,43.88$, $\left.\left.P<0.001, I^{2}=98.6 \%\right)\right)$. But, it has not decreased the heterogeneity. The pooled prevalence of EBF among sample size less than 384 was $68.81 \%(59.45,78.17)$. 


\begin{tabular}{l|rrr} 
Study & & & \\
$\%$ \\
ID
\end{tabular}

FIGURE 3: Forest plot showing the pooled prevalence of replacement feeding practice of HIV-exposed infants in Ethiopia.

\begin{tabular}{|c|c|c|c|}
\hline \multicolumn{2}{|l|}{$\begin{array}{c}\text { Study } \\
\text { ID }\end{array}$} & \multirow{2}{*}{$\begin{array}{c}\text { ES }(95 \% \mathrm{CI}) \\
16.00(13.20,18.80)\end{array}$} & \multirow{2}{*}{$\begin{array}{c}\begin{array}{c}\% \\
\text { weight }\end{array} \\
4.58\end{array}$} \\
\hline Tefera B. and Challi J. (2004) & $* 1$ & & \\
\hline Chernet Hailu (2005) & & $\rightarrow 81.00(77.97,84.03)$ & 4.57 \\
\hline Maru Y and Haidar J (2008) & $\rightarrow-1$ & $15.30(11.40,19.20)$ & 4.56 \\
\hline Muluye et al. (2011) & $\rightarrow$ & $10.50(6.34,14.66)$ & 4.56 \\
\hline Mengstie A. and Tadese T. (2011) & $\longrightarrow$ & $35.60(25.54,45.66)$ & 4.38 \\
\hline Ali Y. and Reddy PS. (2012) & $\leftarrow$ & $11.80(8.53,15.07)$ & 4.57 \\
\hline Wakwoya et al. (2013) & $\rightarrow$ & $14.20(9.96,18.44)$ & 4.55 \\
\hline Girma et al. (2013) & $\rightarrow$ & $6.30(2.99,9.61)$ & 4.57 \\
\hline Modjo KE. and Amanta NW. (2013) & $\rightarrow$ & $34.60(30.13,39.07)$ & 4.55 \\
\hline Bekere et al. (2014) & $\rightarrow$ & $19.50(12.35,26.65)$ & 4.48 \\
\hline Amdom G/Hiwot et al. (2014) & $\rightarrow$ & $5.90(2.78,9.02)$ & 4.57 \\
\hline Tadelech Mebratu (2014) & & $\rightarrow 81.50(77.20,85.80)$ & 4.55 \\
\hline Mengistu and Kedir (2016) & $\rightarrow$ & $15.30(11.40,19.20)$ & 4.56 \\
\hline Demissie et al. (2016) & $\leftarrow$ & $3.70(0.64,6.76)$ & 4.57 \\
\hline Gejo NG.et al. (2016) & + & $6.60(3.81,9.39)$ & 4.58 \\
\hline Ejara et al. (2016) & $\leftarrow$ & $8.30(5.09,11.51)$ & 4.57 \\
\hline Zelalem Ketema (2016) & $\rightarrow$ & $6.30(2.85,9.75)$ & 4.57 \\
\hline Sendo et al. (2017) & $\rightarrow$ & $10.90(6.87,14.93)$ & 4.56 \\
\hline Fikir Tadese (2017) & $\rightarrow$ & $10.90(6.87,14.93)$ & 4.56 \\
\hline Negash et al. (2018) & & $23.30(18.86,27.74)$ & 4.55 \\
\hline Dagnew AB et al. (2018) & $\rightarrow$ & $16.40(11.43,21.37)$ & 4.54 \\
\hline Kefyalew Taye Belete (2019) & & $28.30(19.72,36.88)$ & 4.43 \\
\hline Overall $(\mathrm{I}$-squared $=99.3 \%, \mathrm{p}=0.000)$ & & $20.97(11.35,30.58)$ & 100.00 \\
\hline Note: weights are from random effects analysis & & & \\
\hline
\end{tabular}

FIGURE 4: Forest plot showing the pooled prevalence of mixed feeding practice of HIV-exposed infants in Ethiopia.

\section{Discussion}

We used 26 studies to estimate the pooled prevalence of exclusive breastfeeding among HIV-exposed infants in Ethiopia. Even though WHO recommends exclusive breastfeeding for the first six months of HIV-exposed infants in developing countries, 63.99\% of exposed infants were exclusive breastfed in Ethiopia. This finding is slightly greater than that of national Ethiopian Demographic Health Survey (EDHS) 2016 report of exclusive breastfeeding practices of infants in Ethiopia [46]. This finding also in line with meta-analysis done in Ethiopia among exposed infants 
TABLE 2: Subgroup analysis which describes pooled prevalence of infant feeding practice among HIV-exposed infants in Ethiopia.

\begin{tabular}{|c|c|c|c|c|c|c|}
\hline & Subgroups & & $\begin{array}{l}\text { Number of studies } \\
\text { included }\end{array}$ & $\begin{array}{l}\text { Pooled prevalence } \\
(95 \% \mathrm{CI})\end{array}$ & $P$ value & $I^{2}$ \\
\hline \multirow{10}{*}{$\begin{array}{l}\text { Exclusive breast feeding } \\
\text { practice }\end{array}$} & \multirow{5}{*}{ Region } & Oromia & 6 & $56.56 \%(30.6,86.51)$ & $<0.001$ & $99.5 \%$ \\
\hline & & Addis ababa & 6 & $52.7 \%(32.14,73.25)$ & $<0.001$ & $99.0 \%$ \\
\hline & & Amhara & 7 & $72.84 \%(62.41,83.28)$ & $<0.001$ & $96.8 \%$ \\
\hline & & SNNP & 4 & $54.27(9.63,98.91)$ & $<0.001$ & $99.8 \%$ \\
\hline & & Tigray & 3 & $89.75 \%(87.56,91.95)$ & 0.798 & $0.0 \%$ \\
\hline & \multirow{2}{*}{ Study year } & Before 2015 & 15 & $58.75 \%(42.69,74.82)$ & $<0.001$ & $99.5 \%$ \\
\hline & & 2015 and above & 11 & $71.21 \%(59.19,83.22)$ & $<0.001$ & $98.7 \%$ \\
\hline & \multirow{3}{*}{$\begin{array}{l}\text { Sampling } \\
\text { techniques }\end{array}$} & Random sampling & 6 & $58.25 \%(32.74,83.77)$ & $<0.001$ & $99.3 \%$ \\
\hline & & Consecutive & 4 & $71.26 \%(43.26,99.26)$ & $<0.001$ & $98.7 \%$ \\
\hline & & Systematic random & 15 & $67.40 \%(53.17,81.63)$ & $<0.001$ & $99.3 \%$ \\
\hline \multirow{10}{*}{$\begin{array}{l}\text { Replacement feeding } \\
\text { practice }\end{array}$} & \multirow{5}{*}{ Region } & Oromia & 5 & $13.27 \%(5.75,20.79)$ & $<0.001$ & $98.7 \%$ \\
\hline & & Addis ababa & 4 & $23.80 \%(7.95,39.66)$ & $<0.001$ & $99.2 \%$ \\
\hline & & Amhara & 6 & $16.75 \%(8.50,25.00)$ & $<0.01$ & $96.1 \%$ \\
\hline & & SNNP & 4 & $21.16 \%(-5.05,47.37)$ & $<0.001$ & $99.8 \%$ \\
\hline & & Tigray & 3 & $3.92 \%(2.52,5.33)$ & 0.771 & $0.0 \%$ \\
\hline & \multirow[b]{2}{*}{ Study years } & Before 2015 & 12 & $16.84 \%(10.91,22.76)$ & $<0.001$ & $99.3 \%$ \\
\hline & & $\begin{array}{c}2015 \text { and above } \\
\text { years }\end{array}$ & 10 & $15.37 \%(8.69,22.05)$ & $<0.001$ & $98.0 \%$ \\
\hline & \multirow{3}{*}{$\begin{array}{l}\text { Sampling } \\
\text { techniques }\end{array}$} & Random sampling & 6 & $11.47 \%(4.48,18.46)$ & $<0.001$ & $98.3 \%$ \\
\hline & & Consecutive & 4 & $16.16 \%(2.31,30.01)$ & $<0.001$ & $98.7 \%$ \\
\hline & & Systematic random & 11 & $20.63 \%(10.33,30.93)$ & $<0.001$ & $99.0 \%$ \\
\hline \multirow{10}{*}{ Mixed feeding practice } & \multirow{5}{*}{ Regions } & Oromia & 6 & $26.46 \%(-2.35,55.27)$ & $<0.001$ & $99.7 \%$ \\
\hline & & Addis ababa & 3 & $40.03 \%(-0.80,80.86)$ & $<0.001$ & $99.6 \%$ \\
\hline & & Amhara & 6 & $12.16 \%(10.50,13.82)$ & 0.405 & $1.8 \%$ \\
\hline & & SNNP & 4 & $22.05 \%(8.24,35.87)$ & $<0.001$ & $97.9 \%$ \\
\hline & & Tigray & 3 & $6.29 \%(4.53,8.05)$ & 0.948 & $0.0 \%$ \\
\hline & \multirow{3}{*}{ Study years } & Before 2015 & 10 & $24.46 \%(7.72,41.19)$ & $<0.001$ & $99.5 \%$ \\
\hline & & 2015 and above & 4 & $8.98 \%(4.19,13.77)$ & $<0.001$ & $87.5 \%$ \\
\hline & & Simple random & 6 & $27.52 \%(4.48,50.57 \%)$ & $<0.001$ & $99.4 \%$ \\
\hline & \multirow{2}{*}{ Sampling } & Consecutive & 4 & $14.90 \%(2.40,27.40)$ & $<0.001$ & $97.9 \%$ \\
\hline & & Systematic random & 11 & $13.53 \%(9.96,17.11)$ & $<0.001$ & $89.4 \%$ \\
\hline
\end{tabular}

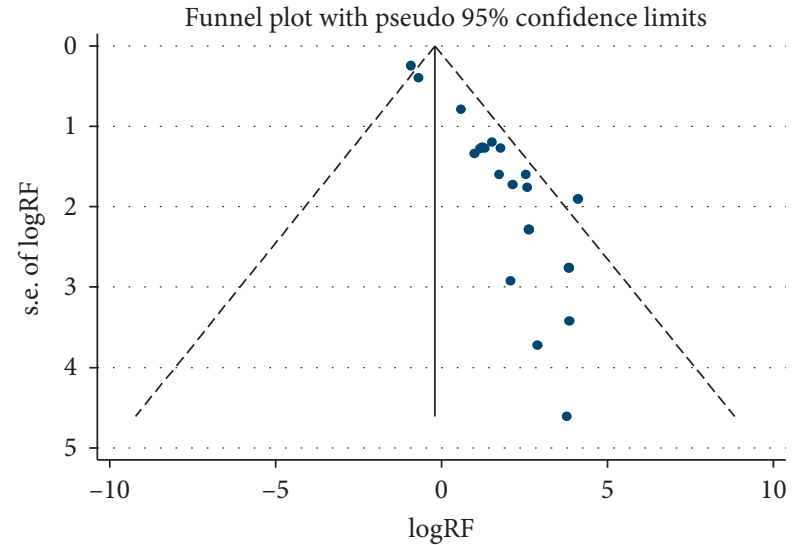

FIGURE 5: Funnel plot for publication bias for the prevalence of replacement feeding practice.

(63.43\%) and the meta-analysis done on exclusive breastfeeding practices in Ethiopia (59.3\%, 59.79\%) [37, 47, 48]. This indicates that there is no significant difference among exposed infant feeding and other infants in Ethiopia which is in line with the World Health Organization recommendation for developing countries [49]. On the other hand, it is greater than that of the study done in Bangladesh (34.5\%),

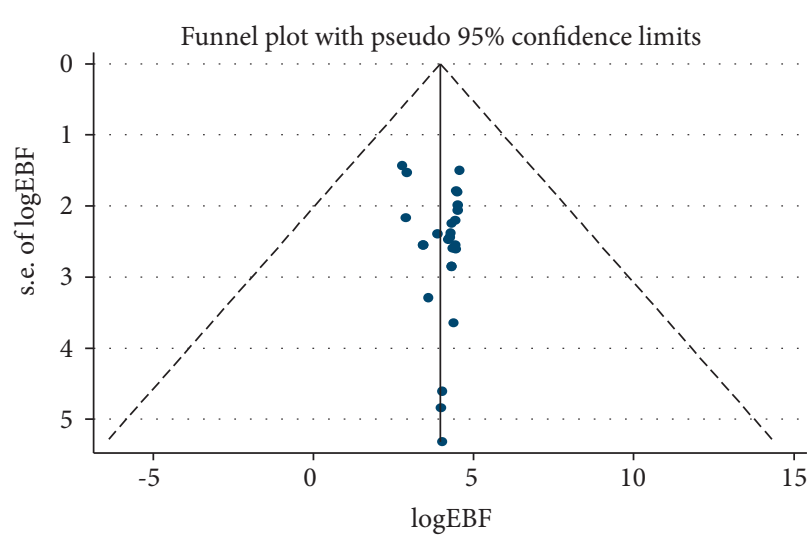

FIgURE 6: Funnel plot for publication bias for the prevalence of exclusive breastfeeding.

Rwanda (43\%), and South Africa (47.9\%) [50-52] but less than that of the study conducted in Kenya (73.7\%) [53]. This difference might be due to difference in the implementation of infant feeding guideline and study design they used.

In this meta-analysis, substantial heterogeneity was observed. The subgroup analysis based on region showed that exclusive breastfeeding practices of HIV-exposed infants were higher in Tigray region followed by Amhara 


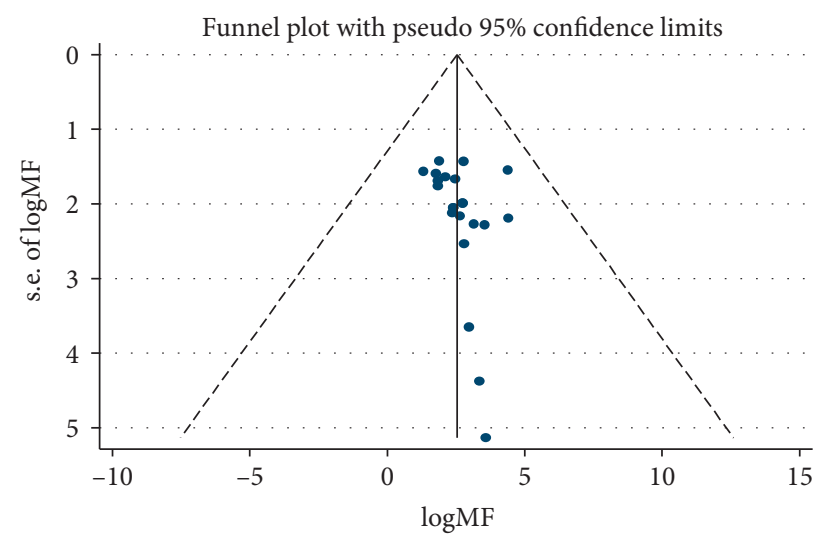

Figure 7: Funnel plot for publication bias for the prevalence of mixed feeding.

TABle 3: Meta-regression to identify source of heterogeneity for the prevalence of infant feeding practices among HIV-exposed infants in Ethiopia.

\begin{tabular}{|c|c|c|c|c|}
\hline Variables & & & Coefficients & $P$ value \\
\hline \multirow{8}{*}{ Exclusive breast feeding practice } & \multirow{2}{*}{ Study year } & 2015 and above & Reference & Reference \\
\hline & & Before 2015 & -12.4204 & 0.221 \\
\hline & \multirow[t]{2}{*}{ Sample } & Sample size & -0.0913904 & 0.007 \\
\hline & & Addis ababa & -37.13872 & 0.039 \\
\hline & \multirow{4}{*}{ Study area(region) } & Amhara & -17.08339 & 0.310 \\
\hline & & Oromia & -31.30989 & 0.077 \\
\hline & & SNNP & -35.57453 & 0.064 \\
\hline & & Tigray & Reference & Reference \\
\hline \multirow{8}{*}{ Replacement feeding practices } & \multirow{2}{*}{ Study years } & 2015 and above & -1.517622 & 0.856 \\
\hline & & Before 2015 & Reference & Reference \\
\hline & \multirow[t]{2}{*}{ Sample size } & Sample size & 0.0266583 & 0.349 \\
\hline & & Addis Ababa & 20.07335 & 0.201 \\
\hline & \multirow{4}{*}{ Study area (regions) } & Amhara & 9.977454 & 0.360 \\
\hline & & Oromia & 14.39511 & 0.498 \\
\hline & & SNNP & 17.26341 & 0.267 \\
\hline & & Tigray & Reference & Reference \\
\hline \multirow{8}{*}{ Mixed feeding practices } & \multirow{2}{*}{ Study years } & 2015 and above & Reference & Reference \\
\hline & & Before 2015 & 14.72224 & 0.111 \\
\hline & \multirow[t]{2}{*}{ Sample size } & Sample size & 0.0581183 & 0.064 \\
\hline & & Addis Ababa & 33.75423 & 0.062 \\
\hline & \multirow{4}{*}{ Study area(regions) } & Amhara & 6.179347 & 0.678 \\
\hline & & Oromia & 20.21536 & 0.185 \\
\hline & & SNNP & 13.25909 & 0.5326 \\
\hline & & Tigray & Reference & Reference \\
\hline
\end{tabular}

region. This may be because these regions have better achievement on infant feeding practices. The pooled prevalence of exclusive breastfeeding among exposed infants increased after 2015 when compared to that before 2015 . This is due to the cumulative effect of MDGs and sustainable development goals.

This meta-analysis also includes 22 studies to estimate the pooled prevalence of replacement feeding practice of HIVexposed infant which was $16.13 \%$. This is lower than that of the study conducted in South Africa (47.3\%) and Rwanda (57 $\%)[51,52]$. This pooled prevalence varies across regions in Ethiopia. Relatively, the highest magnitude was reported in Addis Ababa, and the lowest magnitude was reported in Tigray and Amhara regions. This is because Addis Ababa is relatively urban and has good access to health facility and gets advices on the benefits of replacement feeding. On the other hand, they can afford for replacement feeding. There was no significant difference of replacement feeding practices among exposed infants before and after the periods of 2015 in Ethiopia. This might be because the WHO does not recommend replacement feeding practice in the developing countries including Ethiopia. Therefore, there is no need for special efforts to increase the replacement feeding practice in Ethiopia. Replacement feeding is not recommended for people who do not meet the minimum requirement of the standard stetted by the World Health Organization. Replacement feeding is recommended for Prevention of Mother to Child Transmission (PMTCT) programs in developed countries and also in developing countries if it is acceptable, feasible, affordable, sustainable, and safe [54]. 
Mixed feeding is also common in Ethiopia. The estimated pooled prevalence of mixed feeding practice in Ethiopia among HIV-exposed infant was $20.97 \%$. This finding is slightly lower than that of the study conducted in Ethiopia (23.11\%) [37]. This is because more articles were included to estimate the pooled prevalence of mixed feeding than that of the previous study. This result is also much higher than that of the study performed in South Africa (4.3\%) [52].

The subgroup analysis showed that the pooled prevalence of mixed feeding among HIV-exposed infants varied across the regions of Ethiopia. Addis Ababa has the highest while Tigray region has the lowest pooled prevalence of mixed feeding practices. There was also three times decrement of mixed feeding practices after 2015 in Ethiopia than that before 2015. This is due to the effort made to reduce the risk of HIV transmission through the programs called option $\mathrm{B}^{+}$[55], which was implemented for the last eight years in Ethiopia in different health facilities.

In this meta-analysis, there was publication bias of replacement feeding practices. Even though there was publication bias, there is no significant difference on the estimated pooled prevalence after conducting the trim and fill analysis. And also, there are some individual studies that influence the pooled prevalence of infant feeding practices especially replacement and mixed feeding.

4.1. Limitations of the Study. This review only reports the prevalence of HIV-exposed infant feeding practices in Ethiopia and does not consider different factors contributed to infant feeding practices.

\section{Conclusion and Recommendations}

In Ethiopia, almost three out of five HIV-exposed infants were exclusively breastfed. But still, mixed feeding during the period of 6 months was practiced in almost one-fifth of the exposed infants in Ethiopia. Additionally, replacement feeding was also practiced even though not recommended for developing countries. This indicates that infant feeding practices of HIV-exposed infant in Ethiopia still have a problem and deviated from the WHO recommendation for developing countries. There are regional and time variations among the pooled prevalence of exposed infant feeding practices in Ethiopia. Therefore, special focus will be made to reduce the prevalence of mixed feeding practices, increase the exclusive breastfeeding practices, strengthen the PMTCT service in each health facility, and strictly follow the guidelines for developing countries in each health facility. The government of Ethiopia should strengthen the appropriate exposed infant feeding practices by designating strategies to implement the existing guidelines. Finally, researchers should consider factors that affect infant feeding on the context of HIV-positive mothers.

\section{Abbreviations}

AFASS: Acceptable, feasible, affordable, sustainable, and safe

ART: Anti-Retroviral Treatment
CI: $\quad$ Confidence interval

EDHS: Ethiopian Demographic Health Survey

EBF: $\quad$ Exclusive breastfeeding

HIV: Human immunodeficiency virus

MTCT: Mother-to-child transmission

MDG: Millennium development goals

OR: Odds ratio

PMTCT: Prevention of mother-to-child transmission

WHO: The World Health Organization

SNNP: South Nations, Nationalities, and People's region.

\section{Data Availability}

The data used to support the findings of this study are included within the article.

\section{Conflicts of Interest}

The authors declare that there are no conflicts of interest.

\section{Authors' Contributions}

H. T., T. G., and Y. B were involved in the design, selection of study, data extraction, statistical analysis, and developing the initial drafts of the manuscript. H. T., T. G., A. N., M. D., and D. $\mathrm{H}$ were involved in the quality assessment. H. T and A. N prepared the final draft of the manuscript. W. W and A. A. A. were involved in the editing our manuscript. All the authors read and approved the final draft of the manuscript.

\section{References}

[1] G. Wapang'ana, "Assessment of factors influencing infant feeding practices among HIV positive mothers in Rongo District, Western Kenya," MPH Thesis, School of Public Health Kenyatta University, Rongo, Migori, 2013.

[2] WHO, UNICEF: Guidelines on HIV and Infant Feeding 2010: Principles and Recommendations for Infant Feeding in the Context of HIV and a Summary of Evidence, WHO Library Cataloguing-in-Publication Data, Geneva, Switzerland, 2010.

[3] WHO, Fact Sheet: HIV Treatment and Care: What's New in Infant Diagnosis, WHO Library Cataloguing-in-Publication Data, Geneva, Switzerland, 2015.

[4] World Health Organization, PMTCT Strategic Vision 2010-2015: Preventing Mother-To-Child Transmission of HIV to Reach the UNGASS and Millennium Development Goals, WHO Library Cataloguing-in-Publication Data, Geneva, Switzerland,, 2010.

[5] M. Mahy, J. Stover, K. Kiragu et al., "What will it take to achieve virtual elimination of mother-to-child transmission of HIV? an assessment of current progress and future needs," Sexually Transmitted Infections, vol. 86, no. 2, pp. 48-55, 2010.

[6] C. A. Teasdale, B. J. Marais, and E. J. Abrams, "HIV: prevention of mother-to-child transmission," BMJ Clinical Evidence, vol. 2011, no. 1, 909 pages, 2011.

[7] WHO and UNICEF, Global Strategy for Infant and Young Child Feeding, WHO Library Cataloguing-in-Publication Data, Geneva, Switzerland, 2006.

[8] L. Kuhn and G. Aldrovandi, "Survival and health benefits of breastfeeding versus artificial feeding in infants of HIV-infected women: developing versus developed world," Clinics in Perinatology, vol. 37, no. 4, pp. 843-862, 2010. 
[9] WHO and UNICEF, Planning Guide for National Implementation of the Global Strategy for Infant and Young Child Feeding, WHO Library Cataloguing-in-Publication Data, Geneva, Switzerland, 2007.

[10] World Health Organization, Guideline: Updates On Hiv And Infant Feeding: The Duration Of Breastfeeding, And Support From Health Services To Improve Feeding Practices Among Mothers Living With HIV, WHO Library Cataloguing-inPublication Data, Geneva, Switzerland, 2016.

[11] WHO and UNICEF, A guide on indicators for the monitoring and reporting on the health sector response to HIV/AIDS, World Health organization, Geneva, Switzerland, 2011.

[12] Y. Mengistu and M. Kedir, "Assessment of infant feeding practice among HIV positive mothers and HIV status of their infants in Adama hospital, Oromia regional state, Ethiopia," Journal of Pregnancy and Child Health, vol. 4, no. 346, p. 2, 2016.

[13] Y. Maru and J. Haidar, "Infant feeding practice of HIV positive mothers and its determinants in selected health institutions of Addis Ababa, Ethiopia," Ethiopian Journal of Health Development, vol. 23, no. 2, 2009.

[14] M. Mukerem and J. Haidar, "Assessment of the prevalence and factors influencing adherence to exclusive breast feeding among HIV positive mothers in selected health institution of Addis Ababa, Ethiopia," Ethiopian Journal of Health Development, vol. 26, no. 3, pp. 169-175, 2012.

[15] E. G. Sendo, F. T. Mequanint, and S. GT, "Infant feeding practice and associated factors among HIV positive mothers attending ART clinic in governmental health institutions of bahir dar town, Amhara regional state, Ethiopia," Journal of AIDS and Clinical Research, vol. 9, p. 755, 2017.

[16] M. Z. Demelew and G. Abdeta, "Assessment of exclusive breastfeeding practices among HIV positive women in Addis Ababa," African Journal of Midwifery and Women's Health, vol. 8, no. 1, pp. 14-20, 2014.

[17] D. Muluye, D. Woldeyohannes, M. Gizachew, and M. Tiruneh, "Infant feeding practice and associated factors of HIV positive mothers attending prevention of mother to child transmission and antiretroviral therapy clinics in Gondar Town health institutions, Northwest Ethiopia," BioMed Central Public Health, vol. 12, p. 240, 2012.

[18] H. Genetu, M. K. Yenit, and A. Tariku, "Breastfeeding counseling and support are associated with continuous exclusive breastfeeding from one week to six months of age among HIV exposed infants in north Gondar zone, Ethiopia: a cross-sectional study," International Breastfeeding Journal, vol. 12, p. 21, 2016.

[19] B. Tefera and J. Challi, “Awareness about feeding options for infants born to HIV positive mothers and mother to child transmission of HIV in Gurage zone, South Ethiopia," Ethiopian Journal of Health Development, vol. 21, no. 1, pp. 40-47, 2007.

[20] C. Hailu, Assessment of Knowledge, Attitude \& Practice Among Mothers about Vct and Feeding of Infants Born to Hiv Positive Women in Jimma Town, Ethiopia, Jimma, Ethiopia, 2005.

[21] E. B. Wakwoya, T. A. Zewudie, and K. Z. Gebresilasie, "Infant feeding practice and associated factors among HIV positive mothers in Debre Markos referral hospital east gojam zone, north west Ethiopia," The Pan African Medical Journal, vol. 24, 2016.

[22] Y. Girma, A. Aregay, and G. Biruh, "Infant feeding practice and associated factors among HIV positive mothers enrolled in governmental health facilities in Mekelle town, Tigray region, north Ethiopia," Journal of HIV/AIDS and Infectious Diseases, vol. 2, no. 401, pp. 1-8, 2014.

[23] A. Bekere, W. Garoma, and F. Beyene, "Exclusive breastfeeding knowledge of HIV positive mothers and associated factors in selected health institution of west Oromia, Ethiopia," Universal Journal of Food and Nutrition Science, vol. 2, no. 3, pp. 37-44, 2014.

[24] D. B. Demissie, F. Tadesse, B Bezabih et al., "Infant feeding practice and associated factors of HIV positive mothers attending prevention of mother to child transmission and antiretroviral therapy clinics in shashemene referal hospital," Journal of Health, Medicine and Nursing, vol. 30, 2016.

[25] A. Mengstie and T. Tadese, "Assessment of factors associated with infant and young child feeding practices of human immunodeficiency virus (HIV) positive mothers in selected hospitals of Southern Nations, Nationalities, and Peoples Region (SNNPR) Ethiopia," Journal of AIDS and HIV Research, vol. 8, no. 6, pp. 80-92, 2016.

[26] K. E. Modjo and N. W. Amanta, "Attitude and practice towards exclusive breast feeding and its associated factors among HIV positive mothers in southern Ethiopia," American Journal of Health Research, vol. 3, no. 2, pp. 105-115, 2015.

[27] T. Wondie and B. Worku, "Exclusive breastfeeding and repkacemnt feeding onmorbidityand mortality IN HIV exposed infants at one year age IN tikur anbessa specialized hospital," Ethiopian Journal of Pediatrics and Child Health, vol. 8, no. 8, pp. 52-59, 2012.

[28] G. Hiwot, K. Silassie, G. Mirutse, and H. Alem Desta, "Infant feeding practice of HIV positive mothers and its determinants in public health institutions in central zone, Tigray Region, Northern Ethiopia," International Journal of Pharma Sciences and Research (IJPSR), vol. 23, no. 2, 2014.

[29] S. M. F. Negash and G. Egata, "Infants and young children feeding practice and associated factors among HIV positive mothers of children $0-23$ months in health centers of Gulele sub-city, Addis Ababa, Ethiopia," BMC Research Notes, vol. 12, no. 1, p. 666, 2019.

[30] T. Mebratu, A Resarch Paper Assessement of KAP of HIV Positive Mothers on Vct and Infant Feeding in Akaki Kaliti, (Doctoral Dissertation, Addis Ababa University), Addis Abeba, Ethiiopia, 2014.

[31] A. B. Dagnew, T. Tewabe, A. Birhie et al., "Factors associated with compliance with world health organization-recommended infant-feeding practices by mothers with HIV infection in northwest Ethiopia," Current Therapeutic Research, vol. 91, pp. 39-44, 2019.

[32] D. Ejara, D. Mulualem, and S. Gebremedhin, "Inappropriate infant feeding practices of HIV-positive mothers attending PMTCT services in Oromia regional state, Ethiopia: a crosssectional study," International Breastfeeding Journal, vol. 13, no. 1, p. 37, 2018.

[33] Z. Ketema, Exclusive Breast Feeding Practices and Associated Factors Among HIV Positive Women in Public Health Facilities of Adama Town, Ethiopia, Addis Ababa University, Jimma, Ethiopia, 2016.

[34] F. Tadesse, Infant Feeding Practice and Associated Factors Among HIV Positive Mothers Attending Art Service in Governmental Health Institutions of Bahir Dar Town, Addis Ababa University, Jimma, Ethiopia, 2017.

[35] K. T. Belete, "HIV exposed infants feeding practices and associated factors among mothers attending PMTCT clinics at ambo town public health facilities," Journal of Health and Environmental Research, vol. 6, no. 2, pp. 31-36, 2020. 
[36] N. G. Gejo, H. G. Weldearegay, W. T KT et al., "Exclusive breastfeeding and associated factors among HIV positive mothers in Northern Ethiopia," PloS One, vol. 14, no. 1, e0210782 pages, 2019.

[37] G. M. Belay and C. A. Wubneh, "Infant feeding practices of HIV positive mothers and its association with counseling and HIV disclosure status in Ethiopia: a systematic review and Meta-analysis," AIDS Research and Treatment, vol. 2019, Article ID 3862098, 2019.

[38] M. David, L. Alessandro, T. Jennifer et al., "Preferred reporting items for systematic reviews and meta-analyses: the PRISMA statement," Physical Therapy, vol. 89, no. 9, pp. 873-880, 2009.

[39] Z. Munn, S. Moola, K. Lisy, D. Riitano, and C. Tufanaru, "Methodological guidance for systematic reviews of observational epidemiological studies reporting prevalence and cumulative incidence data," International Journal of EvidenceBased Healthcare, vol. 13, no. 3, pp. 147-153, 2015.

[40] WHO and United Nations Childrens Fund, Guideline: Update on HIV and Infant Feeding: The Duration Ofbreastfeeding, and the Support from Health Services to Improve Feeding Practices Among Mothers Living with HIV, World Health Organisation, Geneva, Switzerland, 2016.

[41] G. Rücker, G. Schwarzer, J. R. Carpenter, and M. Schumacher, "Undue reliance on $\mathrm{I} 2$ in assessing heterogeneity may mislead," BMC Medical Research Methodology, vol. 8, no. 1, p. 79, 2008.

[42] C. B. Begg and M. Mazumdar, "Operating characteristics of a rank correlation test for publication bias," Biometrics, vol. 50, no. 4, pp. 1088-1101, 1994.

[43] M. Egger, G. D. Smith, M. Schneider, and C. Minder, "Bias in meta-analysis detected by a simple, graphical test," British Medical Journal, vol. 315, no. 7109, pp. 629-634, 1997.

[44] S. Duval and R. Tweedie, "Trim and fill: a simple funnel-plot-based method of testing and adjusting for publication bias in metaanalysis," Biometrics, vol. 56, no. 2, pp. 455-463, 2000.

[45] Y. Ali and P. S. Reddy, "IJSR assessment of infant feeding practices among HIV positive mothers receiving ARV/ART and HIV status of their infants with its determinants in South and North Wollo zone, Amhara region, Ethiopia," vol. 4, no. 2, 2015.

[46] ICF and CSACEa, Ethiopia Demographic and Health Survey, Central Statistical Agency, Ethiopia, Rockville, MD, USA, 2016.

[47] A. Alebel, C. Tesma, B. Temesgen, A. Ferede, and G. D. Kibret, "Exclusive breastfeeding practice in Ethiopia and its association with antenatal care and institutional delivery: a systematic review and meta-analysis," International Breastfeeding Journal, vol. 13, p. 31, 2018.

[48] G. E. Wake and Y. M. Mittiku, Prevalence of Exclusive Breastfeeding Practice in Ethiopia and its Association with Maternal Employment: A Systematic Review and MetaAnalysis, 2020, Maternal Employment, Addia Ababa, Ethiopia, 2019.

[49] W. H. Organization, Guidelines on HIV and Infant Feeding 2010: Principles and Recommendations for Infant Feeding in the Context of HIV and a Summary of Evidence, Maternal, Newborn, Child and Adolescent Health, Geneva, Switzerland, 2010.

[50] S. Mihrshahi, N. Ichikawa, M Shuaib et al., "Prevalence of exclusive breastfeeding in Bangladesh and its association with diarrhoea and acute respiratory infection: results of the multiple indicator cluster survey 2003," Journal of Health, Population, and Nutrition, vol. 25, no. 2, p. 195, 2007.
[51] C. A. Peltier, G. F. Ndayisaba, P. Lepage et al., "Breastfeeding with maternal antiretroviral therapy or formula feeding to prevent HIV postnatal mother-to-child transmission in Rwanda," AIDS, vol. 23, no. 18, pp. 2415-2423, 2009.

[52] A. E. Goga, T. Doherty, D. J Jackson et al., "Infant feeding practices at routine PMTCT sites, South Africa: results of a prospective observational study amongst HIV exposed and unexposed infants-birth to 9 months," International Breastfeeding Journal, vol. 7, no. 1, p. 4, 2012.

[53] P. C. Lang'at, I. A. Ogada, A. Steenbeek, G. Odinga, and M. M. Mwachiro, "Do the feeding practices and nutrition status among HIV-exposed infants less than 6 months of age follow the recommended guidelines in Bomet County, Kenya?" BMC Nutrition, vol. 2, no. 1, p. 43, 2016.

[54] C. Evans, C. E. Jones, and A. J. Prendergast, "HIV-exposed, uninfected infants: new global challenges in the era of paediatric HIV elimination," The Lancet Infectious Diseases, vol. 16, no. 6, pp. e92-e107, 2016.

[55] W. P. Update, Use of Antiretroviral Drugs for Treating Pregnant Women and Preventing HIV Infection in Infants, WHO, Geneva, Switzerland, 2012. 